\title{
Révision de la loi sur les produits thérapeutiques Aperçu des propositions de la FMH
}

Gert Printzen ${ }^{a}$,

Hanspeter Kuhn

a Membre du Comité central de la FMH, responsable du domaine Informatique médicale et eHealth

b Avocat, responsable du service juridique de la FMH, secrétaire général adjoint de la FMH
Le Parlement révise actuellement la loi sur les produits thérapeutiques (LPTh). Voici les principales propositions de la FMH à cet égard:

\section{La sécurité des patients suppose un accès simple aux informations relatives aux médicaments}

Le projet de révision ne prévoit qu'à titre facultatif que Swissmedic se charge de l'accès électronique aux informations sur les médicaments [1]. La FMH considère que cette disposition est insuffisante. Pour que les médecins puissent traiter leurs patients de manière sûre, ils doivent pouvoir trouver ces informations de manière simple à un endroit unique, et être certains qu'elles soient exhaustives, à jour, et que leur qualité soit garantie. Ces informations doivent en outre être très bien structurées sous la forme d'une «base de connaissances» (drug dictionary) à proprement parler, afin d'être utilisables dans le cadre de l'e-médication.

\section{L'extension du compassionate use est judicieuse}

La FMH salue la simplification de l'autorisation d'utiliser des médicaments pour traiter des maladies susceptibles d'entraîner la mort ou une invalidité grave. Elle se prononce également clairement en faveur de la simplification du compassionate use au profit d'un cercle déterminé de personnes, en dehors des essais cliniques, et de la mise sur le marché de médicaments autorisés à l'étranger en cas de rupture d'approvisionnement temporaire de médicaments identiques autorisés en Suisse [2].

\section{La promotion des médicaments à usage pédiatrique doit être encouragée, et les femmes enceintes et les seniors pris en compte}

L'extension de la protection conférée par les brevets délivrés pour les médicaments à usage pédiatrique doit être encouragée. Par ailleurs, les déficits grevant la recherche affectent également d'autres groupes dignes de protection, par ex. les femmes enceintes et les seniors. Il convient là aussi de créer des incitatifs pour pouvoir faire bénéficier ces catégories de personnes d'un plus grand nombre de médicaments [3].

Il en va de même en ce qui concerne l'introduction de plans d'investigation pédiatrique pour les nouveaux médicaments - là aussi, les femmes enceintes et les personnes âgées ne doivent pas être oubliées [4].

\section{La remise de médicaments par les médecins relève de la compétence des cantons}

Le Conseil fédéral propose de laisser aux cantons la compétence de réglementer la propharmacie. La FMH adhère à cette proposition, la réglementation au niveau cantonal ayant fait ses preuves. Ces dernières années, les citoyens de divers cantons se sont clairement prononcés en faveur de la propharmacie. Cela permet aux patients de choisir s'ils veulent se faire remettre leurs médicaments dans une pharmacie ou par leur médecin. Compte tenu de leur formation prégraduée et postgraduée, les médecins sont en mesure de gérer la remise des médicaments dans les règles de l'art et le respect des critères commerciaux. Par ailleurs, la compliance des patients se trouve renforcée par le fait que le médecin leur remet directement les médicaments accompagnés des instructions correspondantes pendant ou après la consultation. L'âge ou la maladie diminuent la mobilité de nombreux patients; c'est pour eux un grand soulagement que d'obtenir les médicaments nécessaires de manière directe et sans détours.

\section{Droit des pharmaciens de remettre des médicaments - un assouplissement problématique}

Le projet législatif prévoit que les pharmaciens sont habilités à remettre sans ordonnance médicale certains médicaments spécifiquement désignés de la catégorie de médicaments soumis à ordonnance, et ceci en dehors des cas d'urgence [5]. La FMH considère qu'une telle situation n'est envisageable que si la remise du médicament ne nécessite pas de diagnostic médical de la maladie, si le médicament n'est pas dangereux et si le pharmacien en informe sans délai le médecin traitant.

\section{Pharmaciens et médecins doivent conserver la possibilité de préparer les médicaments}

Le Compendium des médicaments règle de manière appropriée la préparation, à savoir la façon de mettre en place par le biais d'une dilution etc., un médicament déjà fabriqué en vue de l'administrer. Tant les pharmaciens que les médecins ont le droit de procéder à de telles préparations; celles-ci sont indispensables au quotidien dans de nombreuses spécialités médicales telles que l'oncologie, l'anesthésie, la médecine intensive et la dermatologie. La nouvelle formulation de l'art. 7 relatif à la fabrication de médica- 
ments ne doit rien changer au droit des pharmaciens et des médecins en la matière.

\section{L'assurance-qualité doit englober l'ensemble du processus}

Le projet de révision ne prévoit de système d'assurance-qualité qu'en rapport avec la remise de médicaments [6]. Du point de vue de la FMH, une assurance-qualité aussi étroitement limitée n'aurait que peu de sens, car des incidents dus à l'échange de médicaments ou à de faux dosages peuvent survenir du moment de l'établissement de l'ordonnance jusqu'à celui de la prise du médicament; c'est l'ensemble du processus qui est décisif pour la sécurité.

\section{Les médicaments de la médecine complé- mentaire doivent être remis conformément aux règles qui les régissent}

Il doit être possible de remettre des médicaments de la médecine complémentaire si les principes de la thérapie spéciale concernée sont respectés. Le projet de révision actuel ne le permet pas, puisqu'il exige également l'observation des «règles reconnues des sciences pharmaceutiques et médicales» [7].

\section{II n'appartient pas au Conseil fédéral de définir les règles de l'art médical}

Les règles généralement reconnues des sciences et de la pratique médicales ne sont arrêtées ni par le Conseil fédéral, ni par la jurisprudence. Il doit en être ainsi, dans la mesure où ces derniers se fondent sur l'état général des connaissances professionnelles, lequel change dans le temps au gré de l'accroissement des connaissances. Compte tenu du fait qu'il en va de même pour ce qui concerne les sciences médicales et pharmaceutiques, il serait dénué de sens de vouloir les définir dans une ordonnance.

\section{La répercussion des avantages doit être convenue avec les assureurs}

La possibilité que les fournisseurs de prestations conviennent avec les assureurs ou les patients d'une répercussion au pro rata des avantages qui favorise de la manière la plus efficace un approvisionnement des patients en médicaments à prix avantageux [8] doit être encouragée. La FMH approuve l'indication, de manière transparente, des éventuels avantages dans les comptes [9].

\section{Une transparence pertinente en ce qui concerne les mandats d'administrateurs et les participations au sein des entreprises} La FMH considère pertinente la nouvelle obligation prévue par le projet d'informer les patients, sous une forme appropriée, d'éventuels mandats d'administrateurs et fonctions d'experts pour le compte d'entreprises fabriquant ou mettant sur le marché des produits thérapeutiques, ou encore des participations financières importantes à de telles entreprises [10].

\section{Les brevets délivrés pour de simples dosages exigent l'introduction de nouvelles dispositions de protection dans la loi sur les brevets}

Depuis 2010, il est possible de délivrer en Europe des brevets pour de nouveaux dosages sans pour autant que le principe actif ou l'indication ne change. Afin que les médecins puissent continuer à doser et à préparer les médicaments conformément à l'état actuel des connaissances et sans craindre d'être poursuivis pour violation de brevet, il convient d'introduire de nouvelles dispositions protégeant le personnel médical dans la loi sur les brevets [11].

\section{Références}

1 L'art. 67 al. 1 bis du projet de révision stipule que l'institut «peut publier, aux frais du titulaire de l'autorisation, sous forme de liste électronique, les informations destinées aux professionnels et aux patients (informations sur le médicament).»

2 Les art. 9a et 9b, en relation avec l'art. 14 al. 1, prévoient une autorisation simplifiée si cette mesure est compatible avec la protection de la santé, si un grand bénéfice thérapeutique est escompté, et s'il n'existe pas de médicament de substitution et équivalent autorisé en Suisse.

3 Art. $11 \mathrm{~b}$ al. 4.

4 Art. 54.

5 Art. 24 al. 1 bis.

6 Art. 30 al. 2

7 Art. 26 al. 1.

8 Art. 57 a.

9 Art. $57 \mathrm{c}$ al. 1

10 Art. $57 \mathrm{c}$ al. 2.

11 L'art. 9 de la loi sur les brevets, relatif aux «exceptions aux effets du brevet», doit être complété dans ce sens. Le Tribunal fédéral a relevé cette problématique en 2011 (ATF 137 III 170 4A_435-2010 du 4 mars 2011 Merck c. Mepha). 